\title{
REDES SOCIALES Y ESTUDIANTES UNIVERSITARIOS: DEL NATIVO DIGITAL AL INFORMÍVORO SALUDABLE
}

\author{
Gabriel Valerio-Ureña y Ricardo Valenzuela-González
}
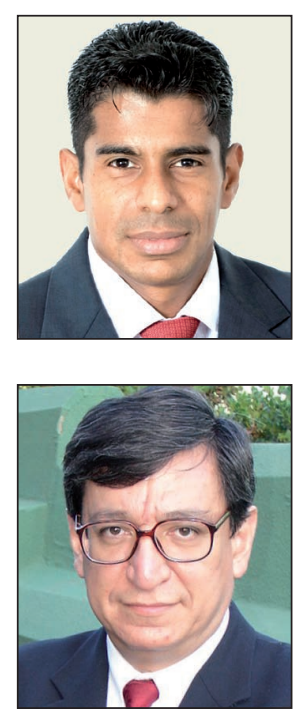

Gabriel Valerio-Ureña tiene estudios de doctorado en innovación educativa por la Universidad Virtual del Tecnológico de Monterrey. Es ingeniero en sistemas computacionales y cuenta con estudios de maestría en administración de tecnologías de información. Ha sido consultor en proyectos de gestión del conocimiento. Es profesor invitado de la Universidad Externado de Colombia y miembro activo de la Comunidad Iberoamericana de Sistemas de Conocimiento.

Tecnológico de Monterrey, Centro de Sistemas de Conocimiento Av. Eugenio Garza Sada, 2501 Sur. Edif. Cetec, Torre norte, piso 3 Col. Tecnológico Monterrey, Nuevo León 64849, México gvalerio@itesm.mx

Ricardo Valenzuela-González tiene estudios de doctorado en psicología educativa por la Universidad de Texas en Austin (EUA). Es miembro del claustro del programa de doctorado en innovación educativa del Tecnológico de Monterrey, del cual fue también su director. Fue miembro del Sistema Nacional de Investigadores del Consejo Nacional de Ciencia y Tecnología (Conacyt), siendo el e-learning una de sus líneas de investigación.

Tecnológico de Monterrey, Universidad Virtual, Escuela de Graduados en Educación Av. Eugenio Garza Sada, 2501 Sur, Edif. Cedes, sótano 1 Col. Tecnológico Monterrey, Nuevo León 64849, México jrvg@itesm.mx

\section{Resumen}

Las redes sociales en línea son una de las herramientas tecnológicas más utilizadas actualmente por los estudiantes universitarios, y asimismo algunas empresas empiezan a hacer uso de ellas para soportar sus procesos de negocio. Sin embargo, una parte de la sociedad tiene la impresión de que son sólo una pérdida de tiempo. Algunas investigaciones demuestran que bien utilizadas pueden traer grandes beneficios como: la adquisición de competencias informáticas, el acceso a repositorios de información y el desarrollo del capital social. Ser un nativo digital no garantiza que los estudiantes universitarios serán capaces de obtener estos beneficios. Los profesores y las universidades en general, más que bloquear el acceso de los estudiantes a estas redes, deben preocuparse por desarrollar las competencias informáticas necesarias para producir informívoros saludables.

\section{Palabras clave}

Redes sociales, Estudiantes universitarios, Competencias informáticas, Informívoros saludables, Nativos digitales.

Title: Social networks and university students. From digital native to healthy informivore

\begin{abstract}
Online social networks are one of the technological tools most used by university students at present. Likewise, some enterprises are beginning to use them to support their business process. However, one part of the society has the impression that these are just a waste of time. Despite this, studies have shown that, properly used, social networks can have great benefits, such as the development of information literacy, access to information repositories and the development of social capital. Being a digital native does not guarantee that university students will be able to obtain these benefits. Teachers and universities in general, rather than block student access to these networks, must concern themselves with developing the necessary information literacy to produce healthy informivores.
\end{abstract}

\section{Keywords}

Social networks, University students, Information literacy, Healthy informivores, Digital natives.

Valerio-Ureña, Gabriel; Valenzuela-González, Ricardo. "Redes sociales y estudiantes universitarios: del nativo digital al informívoro saludable". El profesional de la información, 2011, noviembre-diciembre, v. 20, n. 6, pp. 667-670. 


\section{Introducción}

La evolución de la sociedad no se detiene. Los clásicos gritos de los padres de los ochenta pidiendo que sus hijos adolescentes colgaran el teléfono porque monopolizaban el medio de comunicación familiar, han evolucionado. Ahora el padre, que era el adolescente que se pasaba horas pegado al teléfono, pide a su hijo que apague el ordenador preguntando con sincera curiosidad: "¿no te aburres de pasarte todo el día en eso?". Los jóvenes de hoy no son los mismos que los de ayer, no se visten, bailan, piensan, actúan y se comunican igual; y sin embargo, en forma similar a los jóvenes de antes, los actuales también aprenden mediante actividades lúdicas cómo aprovechar su entorno para triunfar en la vida en sociedad (Sánchez-Navarro; Aranda, 2011).

Los medios impresos, la radio, la televisión, la telefonía móvil y por supuesto internet, han favorecido el surgimiento de millones de fuentes de información en toda la Tierra. El mundo de hoy está regido por la información; todos los días creamos y consumimos grandes cantidades gracias a los avances tecnológicos. Si bien se tiene claro que no todo el mundo tiene acceso a internet, y en ocasiones ni siquiera a los servicios básicos, no se puede negar que su presencia, en la mayoría de las poblaciones urbanas, ha transformado el quehacer cotidiano.

Estos cambios tecnológicos han venido necesariamente acompañados de un cambio en la perspectiva sociocultural. Parte de ellos están ligados a la nueva mentalidad de los jóvenes y cómo éstos están mucho más abiertos al cambio y tienen una mayor cantidad de hábitos relacionados con la comunicación con otros. El sistema educativo no es ajeno a estos cambios, ya que de alguna manera se ha visto en la necesidad de evolucionar para responder a las exigencias de los nuevos estudiantes y adaptarse a las nuevas condiciones y a las nuevas tecnologías. Estos ámbitos tecnológicos y sociales han reformado la visión que los estudiantes y los profesores tenían del aprendizaje.

Los jóvenes de hoy no son los mismos que los de ayer pero también aprenden cómo aprovechar su entorno para triunfar en la vida en sociedad mediante actividades lúdicas

\section{Impacto de las redes sociales}

Los nuevos estudiantes, que pertenecen a la generación Red, redefinen la forma en la que quieren interactuar y aprender. Un cierto grado de alfabetización digital, el acceso a recursos electrónicos e internet, y la necesidad imperiosa de mantenerse comunicados, son condiciones que les permiten participar de manera más interactiva. Gracias a que muchos de ellos cumplen en gran medida las condiciones antes mencionadas, hemos sido testigos del boom de las redes sociales en línea y del software social en general. Servicios como Facebook, Twitter, MySpace y YouTube entre otros, han permitido que las personas se mantengan en completa interacción de manera barata, sencilla y entretenida.
Hoy, como seguramente ha sucedido siempre, se suele culpar a las nuevas tecnologías. Las universidades hacen estudios que muestran una relación entre el uso de redes sociales y el bajo nivel académico; la policía señala que el crimen organizado utiliza estas redes como bancos de información para identificar futuras víctimas; y algunos padres terminan concluyendo que las redes sociales en línea son el demonio en persona o, por lo menos, la reencarnación del Big brother de George Orwell. Sin embargo, también existen investigaciones científicas relacionadas con el conectivismo que muestran resultados con un impacto positivo del uso de redes sociales en línea (Ellison; Steinfield; Lampe, 2007; Holder, 2006; Ito et al., 2008), destacando la adquisición de competencias informáticas, el desarrollo del capital social y el incremento de fuentes fiables de información.

\section{Las redes sociales en línea permiten que un usuario administre no sólo contactos, sino potenciales fuentes de información}

Es verdad que el mal uso de esos sistemas puede causar distracción, pérdida de tiempo y de privacidad, entre otros aspectos negativos, pero bien utilizadas pueden traer beneficios importantes a cualquier persona, incluyendo los estudiantes universitarios. A raíz de una investigación webnográfica realizada a través de Facebook (Valerio-Ureña, 2010a) se concluye que hay impactos positivos de las redes sociales en línea en los estudiantes universitarios. Dichas redes ayudan a:

- Adquirir competencias informáticas que serán útiles a los estudiantes universitarios no sólo para aprender, sino quizás para ser profesionales más eficientes. Aprenden la lógica de la web 2.0, desarrollan las habilidades necesarias para editar y subir fotos y vídeos a la Red, aprenden a usar la tecnología móvil, y usan blogs, entre otras cosas. Muchos de estos aprendizajes los pueden adquirir por motivaciones como conversar con algún amigo en la red social, o crear un perfil más sofisticado, pero en cualquier caso lo importante es que adquieren las competencias.

- Desarrollar el capital social. Un elemento clave para compartir el conocimiento es la confianza. A mayor confianza mayor transferencia de conocimiento. El uso de las redes sociales en línea favorece el desarrollo del capital social y por consiguiente la confianza y la transferencia de conocimiento. A diferencia de una plataforma tradicional de aprendizaje, las redes sociales en línea permiten la interacción constante en un ambiente informal y relajado, así como el acceso a información personal del contacto.

- Administrar repositorios de información. Los contactos de una red social, según la teoría del conectivismo propuesta por Siemens (Siemens, 2004; Siemens; Tittenberger, 2009), representan potenciales repositorios de información para alcanzar el aprendizaje. Permiten que un usuario administre no sólo contactos, sino potenciales fuentes de información. Mediante ellas se pueden hacer listas de usuarios en base a criterios, que permitan discernir qué contactos pueden ser fuentes de información fiables ante un requerimiento de aprendizaje. 
La realidad es que, nos guste o no, los estudiantes universitarios hacen uso de las redes sociales en línea. De acuerdo con investigaciones como la de Ellison, Steinfield y Lampe (2007) y Valerio-Ureña (2010a), en ciertos contextos, más del $90 \%$ de los estudiantes universitarios pertenecen a alguna red social en línea. Aunque a algunos padres y profesores les puede parecer una pérdida de tiempo, quizás estas herramientas los están preparando para el futuro que les espera como profesionales. De hecho, de acuerdo con Levy (2009), el término "empresa 2.0 " se refiere a la implementación de la web 2.0 en la infraestructura tecnológica de las empresas y según esta autora existen muchas empresas que ya son 2.0. En este sentido, Havenstein (2007) habla sobre una investigación de Forrester que indica que 106 de 119 compañías con más de 500 empleados utilizan alguna herramienta web 2.0 como blogs, wikis, podcasts, rss, redes sociales o etiquetado social. Por su parte Schneckenberg (2009) señala que una encuesta de Mckinsey encontró que el $75 \%$ de los directivos encuestados han invertido o plantean invertir más en herramientas web 2.0. Dada esta situación es muy probable que los estudiantes universitarios de hoy trabajen mañana en empresas 2.0.

El papel de los profesores y de las universidades no es bloquear a los alumnos el acceso a las redes sociales, sino entender su naturaleza para encontrar la manera de sacarles el máximo provecho

\section{Informívoros sanos, no sólo nativos digitales}

Las redes sociales en línea son una más de las múltiples herramientas que hoy están al alcance de todos. "Sin embargo, ninguna herramienta es por sí misma positiva o negativa. Es la forma en que se utiliza lo que hará la diferencia entre un resultado positivo y uno negativo" (Valerio-Ureña, 2010b).

El papel de los profesores y las universidades no es bloquear a los alumnos el acceso a las redes sociales, sino entender su naturaleza para encontrar la manera de sacarles el máximo provecho. En todo caso, como instituciones educativas, deben preocuparse por desarrollar las competencias informáticas necesarias para que los universitarios aprovechen de la mejor manera estas aplicaciones. Incluyendo aquellas competencias que les permitan disminuir los riesgos asociados a su uso, por ejemplo en términos de seguridad y privacidad de la información. Debemos asegurarnos de que nuestros estudiantes no sean sólo nativos digitales sino que sean informívoros saludables.

George Miller propuso el término Informivore para definir a la persona que consume información. Su traducción al español sería algo parecido a informívoro. Evidentemente el término tiene una analogía con herbívoros, omnívoros y carnívoros. Hoy existen los vegetarianos puros, están los que no comen carnes rojas y también los que adoran comerlas. Cada uno de ellos puede decir que lo que come es lo que necesita el cuerpo humano. La polémica puede existir, pero pocos podrán discutir que gran parte de los que viven en nuestra sociedad, aquella dominada por internet y las redes sociales en línea, es un informívoro: un ser que procesa información. Bajo esta definición está claro que no es el hombre moderno, el de la sociedad basada en conocimiento, el único que ha sido un informívoro; desde sus más lejanos orígenes, la humanidad siempre ha estado formada por informívoros, siempre hemos hecho uso de la información para tomar decisiones.

Podemos hablar hoy de un informívoro tan sólo por la cantidad de información a la que se accede. Si bien siempre hemos hecho uso de ella, nadie podrá negar que hoy gracias a internet, las redes sociales, los móviles y otros avances de las TIC, tenemos acceso a mucha más información y mucho más rápido que hace tan sólo 10 años. Llamamos informívoro al que consume (procesa) información en grandes cantidades, como hoy llamamos, de manera informal, carnívoro al que come demasiada carne.

En World wide words se indica que la analogía lingüística entre informívoro y carnívoro no es de lo más correcta pues el latín vorare, significa devorar, y la información no se devora. Es precisamente esta diferencia lo que hace de la información un recurso tan valioso. Cuando alguien devora una manzana, por hambre o por gula, otro pierde la oportunidad de comerse esa manzana. Sin embargo, cuando alguien lee un libro (que contiene información) hay posibilidades de que cientos o miles de personas lean también ese libro. Con la llegada de internet y la explosión de las redes sociales, la información en formato digital puede llegar a millones de personas sin que sea devorada por ninguno de sus lectores. En gran parte gracias a esto es por lo que nos hemos convertido en informívoros. Sin embargo, así como un carnívoro o un vegetariano pueden enfermar por la comida que consumen, un informívoro puede enfermar por la información que consume. Las redes sociales en línea pueden ser una excelente herramienta para crear y transferir información, sin embargo nadie verifica que esa información sea sana, sea fiable. Sacarle provecho a estas herramientas depende en gran medida de las competencias informáticas de quienes las utilizan.

\section{http://www.worldwidewords.org}

A finales de la década de los 90 y principios del siglo XXI, organismos como el Banco Mundial, la Comisión Europea, la $O E C D$ y las Naciones Unidas comenzaron a hablar de la necesidad de desarrollar competencias informáticas en los trabajadores de la sociedad basada en conocimiento. Lo que la sociedad reclama es tener más informívoros, pero no de cualquier tipo. Así como hay frutas con más vitaminas y carnes con más proteínas, también hay diferencias en la calidad de la información que se consume. Un informívoro puede ser capaz de acceder a un gran volumen de información utilizando las redes sociales en línea. Sin embargo, no necesariamente por eso será capaz de sacarle provecho.

Las universidades deben preparar hoy a los profesionales de mañana, pero las exigencias que tendrán no son las mismas que las que se tuvieron ayer. Los profesionales de mañana, los trabajadores del conocimiento, tendrán que ser personas capaces de desenvolverse de manera eficiente en un ambiente rico en tecnologías de información, y seguramente usando redes sociales en línea. Preparar profesionales que son capaces de acceder a grandes volúmenes de informa- 


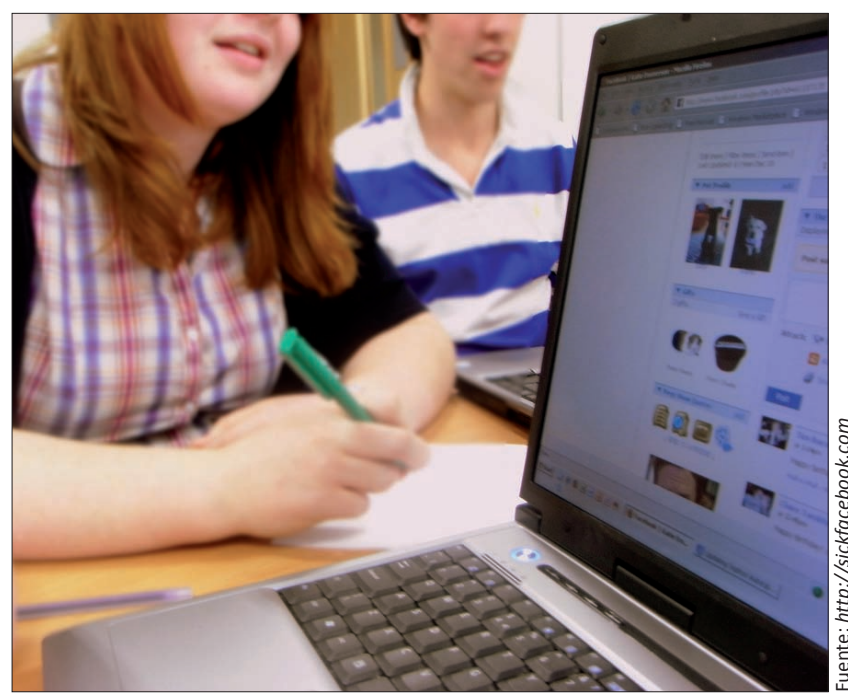

La información es el alimento del trabajador del conocimiento, del informívoro

ción pero no de sacarle provecho a ésta y estar tranquilos, sería tan irresponsable como una madre que cree que tener un niño obeso es sano.

\section{Conclusión}

La información es el alimento del trabajador del conocimiento, del informívoro. Las universidades deben darse cuenta de que no porque los estudiantes sean informívoros están saludables. Los estudiantes deben ser capaces de acceder a la información como un requisito básico para no sufrir inanición, pero también deben saber qué información les conviene consumir y cuál no, o al menos en qué cantidades, para no tener problemas de obesidad o de anemia informativa, por ejemplo al consumir información chatarra. Es decir, deben ser capaces de evaluar y organizar la información que consumen.

Sin embargo el futuro trabajador del conocimiento no puede conformarse con acceder, evaluar y organizar información para utilizarla. Debe ser capaz de crear su propia información y compartirla con quien la necesita, tanto como cualquier ciudadano comprometido con su sociedad debe ocuparse no sólo de saciar su hambre, sino de ser productivo y compartir el alimento con quienes lo necesitan.

Aunque para muchas personas la participación en las redes sociales les puede parecer una pérdida de tiempo, es probable que con ayuda de los profesores, estas herramientas ayuden a preparar mejor a los estudiantes universitarios para desempeñarse en la sociedad basada en conocimiento. Una sociedad donde la información debe fluir como la sangre, siendo las redes sociales sus venas. Desarrollar las competencias informáticas de los alumnos reducirá sin duda la posibilidad de que estas venas no lleven información suficiente o se obstruyan por consumir información chatarra.

\section{Bibliografía}

Ellison, Nicole; Steinfield, Charles; Lampe, Cliff. "The benefits of Facebook 'friends': social capital and college students' use of online social network sites". Journal of computer-mediated communication, 2007, v. 12, n. 4.

http://jcmc.indiana.edu/vol12/issue4/ellison.html http://dx.doi.org/10.1111\%2Fj.1083-6101.2007.00367.x

Havenstein, Heather. "CIOs use web 2.0 to keep up with competition: study". Computer world, 26 marzo 2007. http://computerworld.co.nz/news.nsf/mgmt/F3CF939BD89 D1620CC2572A70007CBC6

Holder, Daniel. Ethnographic study of the effects of Facebook.com on interpersonal relationships. University of Chicago, 2006.

http://www.docstoc.com/docs/2374240/EthnographicStudy-of-the-Effects-of-Facebookcom-on-Interpersonal

Ito, Mizuko; Horst, Heather; Bittani, Matteo; Boyd, Danah; Herr-Stephenson, Becky; Lange, Patricia; Pascoe, C. J.; Robinson, Laura. Living and learning with new media: summary of findings from the Digital Youth Project. The John D. and Catherine T. MacArthur Fundation, 2008.

http://www. macfound.org/atf/cf/\%7BB0386CE3-8B294162-8098-E466FB856794\%7D/DML_ETHNOG_WHITEPAPER.PDF

Levy, Moria. "Web 2.0 implications on knowlegde management". Journal of knowledge management, 2009, v. 13, n. 1, pp. 120-134. http://lpis.csd.auth.gr/mtpx/km/material/JKM-13-1d.pdf http://dx.doi.org/10.1108\%2F13673270910931215

Sánchez-Navarro, Jordi; Aranda, Daniel. "Internet como fuente de información para la vida cotidiana de los jóvenes españoles". El profesional de la información, 2011, enerofebrero, v. 20, n. 1, pp. 32-37.

http://eprints.rclis.org/bitstream/10760/15332/1/032-037. $p d f$

http://dx.doi.org/10.3145\%2Fepi.2011.ene.04

Schneckenberg, Dirk. "Web 2.0 and the empowerment of the knowledge worker". Journal of knowledge management, 2009, v. 13, n. 6, pp. 509-520.

http://dx.doi.org/10.1108/13673270910997150.

Siemens, George. "Connectivism: a learning theory for the digital age". Elearnspace everything elearning, 12 Dec. 2004.

http://www.elearnspace.org/Articles/connectivism.htm

Siemens, George; Tittenberger, Peter. Handbook of emerging technologies for learning, 2009.

http://umanitoba.ca/learning_technologies/cetl/HETL.pdf

Valerio-Ureña, Gabriel. E-learning 2.0 y el impacto de las redes sociales en línea en los estudiantes universitarios. Tesis doctoral, Universidad Virtual, Tecnológico de Monterrey, México, 2010a.

http://www.gabrielvalerio.com/ensayos/tesis_doctoral_ gv.pdf

Valerio-Ureña, Gabriel. "E-learning 2.0 y el impacto de las redes sociales en línea en los estudiantes universitarios". Transferencia, 2010b, v. 23, n. 91, pp. 24-25.

http://www.gabrielvalerio.com/ensayos/tesis_transferen cia_GVU_2010.pdf 